\title{
EBM-Reform
}

\section{Operation ohne Nebenwirkung}

\author{
Stärkere Morbiditätsorientierung in der Vergütung, Aktualisierung des \\ kalkulatorischen Arztlohns und der Praxiskosten - das sind Ziele einer \\ behutsamen EBM-Reform.
}

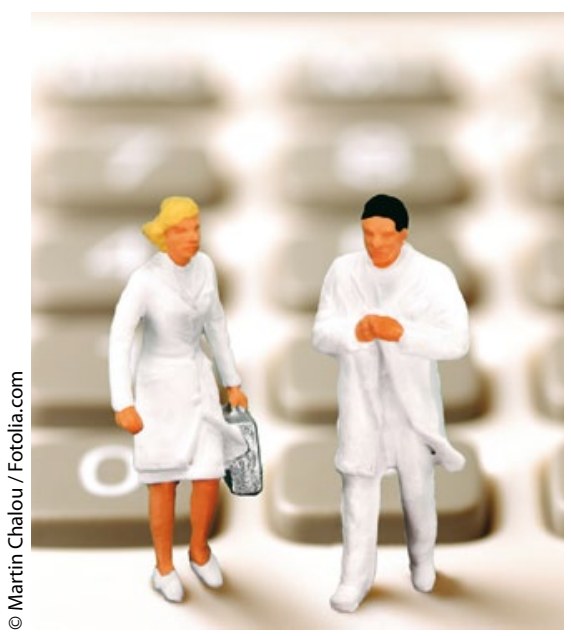

$\longrightarrow$ ie KBV will aus Fehlern klug werden. Trotz erheblicher Zuwächse bei der Gesamtvergütung in den Jahren 2007 bis 2009 löste die letzte große EBM-Reform erheblichen Verdruss aus, weil es zu Verwerfungen zwischen und auch innerhalb von Arztgruppen kam, und als Folge dessen zu erneuten Korrekturläufen - mitunter im Quartalstakt. „Deshalb wollen wir keine radikale Reform, sondern eine schrittweise Evolution", versichert KBV-Vorstandsvorsitzender Dr. Andreas Köhler. Das heißt: Es wird keine Änderung der Kapitelstruktur geben. Regelhafte Leistungen werden weiterhin in Komplexen vergütet.

Alle EBM-Kapitel werden einzeln überprüft und gegebenenfalls überarbeitet. Ursächlich dafür ist der gesetzliche Anspruch auf eine Vergütung und ihre Weiterentwicklung nach der Morbidität und dem daraus resultierenden Behandlungsbedarf. Widersprüchlich ist der gegenwärtig hohe Pauschalisierungsgrad der Vergütung, der es schwer macht, Leistungsverlagerungen von der stationären in die ambulante Behandlung nachzuweisen.
Trennung der Vergütung zwischen Haus- und Fachärzten soll bleiben

Eine neue Kategorie in der Vergütungssystematik sollen Zeitzuschläge für Leistungen bei besonderer Inanspruchnahme durch chronisch kranke Patienten sein.

Die bisherige Trennung der Vergütung von Haus- und Fachärzten bleibt erhalten, so versichert Köhler. Nach einem Beschluss der KBV-Vertreterversammlung vom 26. April soll der Vorstand mithilfe externer Gutachter vor dem Hintergrund der aktuellen Gesetzeslage und der höchstrichterlichen Rechtsprechung das aktuelle Verfahren der Vergütungstrennung und die Vorwegabzüge für bestimmte Leistungen bis Ende September überprüfen lassen. Die FALK-KVen sehen dies mit Besorgnis.

Notwendig ist laut Köhler aber auch eine Überprüfung der Leistungsbewertung. Eine wesentliche Einflussgröße ist der kalkulatorische Arztlohn, der sich nach dem Gehalt eines Klinikoberarztes bemessen soll. Das sind derzeit 105.000 EUR im Jahr. Dieser kalkulatorische Arztlohn stammt aber aus den Gehaltstarifen der Klinikärzte von 2004 - jener Zeit, in der der Marburger Bund noch unter Federführung von Verdi die Tarifgehälter der Ärzte verhandeln ließ. Seit der Marburger Bund als Spartengewerkschaft eigenständig Tarifverträge abschließt, sind die Klinikgehälter kräftig gestiegen. Daran mit dem kalkulatorischen Lohn für Vertragsärzte anzuknüpfen wird umso anspruchsvoller, als nicht einmal der Wert von 2004 von den meisten Arztgruppen erreicht wird.

Der zweite, ebenfalls konfliktreiche Aspekt sind die Praxiskosten. Die Kalkulationsbasis für die geltende Vergütung ist zehn Jahre alt, die seitherige Inflation ist nicht berücksichtigt. Nach Erkenntnissen der KBV haben die Ärzte darauf mit Personalabbau und Rückstellung von
Investitionen reagiert. Köhler beziffert den Investitionsrückstau in den Praxen auf bis zu 3 Milliarden EUR. Besonders prekär erscheint aber im Lichte knapp werdender ärztlicher Arbeitskraft, dass die Vertragsärzte bei ihren medizinischen Fachangestellten sparen (müssen). Dies ist ein Widerspruch zu der als notwendig anerkannten Delegation ärztlicher Leistungen an andere medizinische Fachberufe.

\section{"Orientierungspunktwert"-Modelle}

Ein ebenfalls schwer zu erreichendes Verhandlungsziel der KBV wird sein, dass der vereinbarte Orientierungspunktwert auch dem kalkulatorischen Punktwert entspricht und idealerweise bei 6 Cent liegt. Köhler weiß, dass dies ceteris paribus ein zusätzliches Vergütungsvolumen von 17 Milliarden EUR erfordern würde, was jenseits jeder wirtschaftlichen Realität liegt. Eine Möglichkeit: Die Punktzahlen je Leistung bei gleichem Preis so absenken, dass der erwünschte Orientierungspunktwert erreicht wird. Das kann man freilich nicht über alle Leistungen gleichmäßig machen. Eine Option wäre aber, die Zeitbedarfkalkulation differenziert neu zu justieren und dabei auf Kosten technischer zugunsten zeitgebundener Leistungen Honorar freizuschaufeln. Eine weitere, durchaus erwägenswerte Möglichkeit wäre es, die Fixkosten einer Praxis nur bis zu einer bestimmten Fallzahl pro Praxis oder Arzt zu vergüten und darüber hinaus nur noch die variablen Kosten zu honorieren. Köhler gesteht jedoch zu, dass es nicht einfach ist, die jeweiligen Grenzwerte festzulegen. Das müsse auf jeden Fall differenziert geschehen. Dabei sollten auch die Länder-KVen Gestaltungsspielraum haben: bei besonders förderungswürdigen Praxen und Leistungen, unter Sicherstellungsaspekten und unter Berücksichtigung besonderer Praxisstrukturen. Dieses Modell würde es nach Köhlers Auffassung erlauben, auf die rund 22.000 Leistungsausschlüsse im EBM zu verzichten.

Helmut Laschet 